\title{
OBSERVABLE CONSEQUENCES OF MERGER-DRIVEN GAPS AND HOLES IN BLACK HOLE ACCRETION DISKS
}

\author{
Kayhan GÜLtekin and Jon M. Millë̈ \\ Department of Astronomy, University of Michigan, 500 Church Street, Ann Arbor, MI 48109, USA; kayhan@umich.edu, jonmm@umich.edu \\ Received 2012 July 2; accepted 2012 October 29; published 2012 November 28
}

\begin{abstract}
We calculate the observable signature of a black hole $(\mathrm{BH})$ accretion disk with a gap or a hole created by a secondary $\mathrm{BH}$ embedded in the disk. We find that for an interesting range of parameters of $\mathrm{BH}$ masses $\left(\sim 10^{6}-10^{9} M_{\odot}\right)$, orbital separation ( $\sim 1$ AU to $\sim 0.1 \mathrm{pc})$, and gap width (10-190 disk scale heights), the missing thermal emission from a gap manifests itself in an observable decrement in the spectral energy distribution (SED). We present observational diagnostics in terms of power-law forms that can be fit to line-free regions in active galactic nucleus (AGN) spectra or in fluxes from sequences of broad filters. Most interestingly, the change in slope in the broken power law is almost entirely dependent on the width of the gap in the accretion disk, which in turn is uniquely determined by the mass ratio of the BHs, such that it scales roughly as $q^{5 / 12}$. Thus, one can use spectral observations of the continuum of bright AGNs to infer not only the presence of a closely separated BH binary, but also the mass ratio. When the BH merger opens an entire hole (or cavity) in the inner disk, the broadband SED of the AGNs or quasar may serve as a diagnostic. Such sources should be especially luminous in optical bands but intrinsically faint in X-rays (i.e., not merely obscured). We briefly note that viable candidates may have already been identified, though extant detailed modeling of those with high-quality data have not yet revealed an inner cavity.
\end{abstract}

Key words: black hole physics - galaxies: active

\section{INTRODUCTION}

The prevalence of supermassive black holes (SMBHs) at the centers of galaxies is well established (Richstone et al. 1998). In the context of a hierarchical merging universe, merging galaxies can lead to the merging of the black holes (BHs; Volonteri et al. 2003). Mergers of BHs are important as one potential pathway for the growth of BHs and have even been suggested as the principal cause of the scaling relations between $\mathrm{BH}$ mass and host galaxy properties (Jahnke \& Macciò 2011). Mergers are also strong gravitational wave emitters, and the asymmetric emission of gravitational waves, especially from spinning $\mathrm{BHs}$, can lead to a recoil of the merged BH large enough to kick it out of the host galaxy, potentially influencing the $\mathrm{BH}$ occupation fraction of galaxies (e.g., Volonteri et al. 2008; Baker et al. 2008; Merritt et al. 2004). Even if two galaxies with BHs merge and the BHs sink to the center of the merged galaxy, however, it is not a given that the BHs will merge within a Hubble time (e.g., Milosavljević \& Merritt 2003; Begelman et al. 1980).

The search for precursors to $\mathrm{BH}$ mergers has naturally focused on active galactic nuclei (AGNs) that are either spatially resolvable at separations of $\sim 1 \mathrm{kpc}$ (e.g., Comerford et al. 2012; Blecha et al. 2012) or that have spectroscopically distinct broad line regions at separations of $\sim 0.1$ pc (e.g., Gaskell 1996; Boroson \& Lauer 2009; Smith et al. 2010; Eracleous et al. 2011).

One avenue for finding BH pairs at very small separations comes from an analogy with protoplanetary disks in which the presence of a planet may be inferred from its influence on the protoplanetary disk, particularly through gaps and holes in the disk carved out by the planet (Goldreich \& Tremaine 1980; Takeuchi et al. 1996; Armitage \& Rice 2005; Espaillat et al. 2008). Here, we consider the observable consequences of an accretion disk gap caused by a secondary $\mathrm{BH}$ as has been considered before (Syer et al. 1991; Haiman et al. 2009; Chang et al. 2010). Owing to the complexity of AGNs and quasar spectra, we have developed observational diagnostics in terms of power-law forms that can be fit to line-free regions in spectra or potentially even to fluxes derived from sequences of broad filters. We have also considered signatures that may be evident in the broadband spectral energy distributions (SEDs) of quasars and AGNs that span the optical and X-ray regimes.

In Section 2, we describe our assumptions regarding the accretion disk and secondary BH in which we are interested. Then, we outline the conditions for a gap to be opened and its subsequent evolution in Sections 3 and 4, respectively. In Section 5, we present our calculations of the SED from a gapped accretion disk and the physical inferences that can be made from observations. In Section 6, we describe the basic electromagnetic appearance of an accretion disk with a large inner hole and suggest some candidate sources. Finally, we discuss our results and future work in Section 7.

\section{DESCRIPTION OF THE PHYSICAL SYSTEM}

The presence of a (proto)planet in a protoplanetary disk in orbit around a central star can lead to the transfer of angular momentum such that the planet moves inward (Type I migration; Goldreich \& Tremaine 1980). Under certain circumstances (Section 3), the planet can clear material near its radius, opening up a gap. Dynamical interactions between the planet and material at the gap edges tend to drive both the gap and the planet inward (type II migration; Takeuchi et al. 1996).

We are interested in the observational consequences of analogous systems for SMBHs. Consider a central BH of mass $M$ with an accretion disk around it with a secondary BH of mass $m_{s}$, mass ratio $q \equiv m_{s} / M$, in the plane of the disk at an orbital radius of $r$. If the secondary BH can open a gap in the accretion disk, then the relative difference in emission would create an observational signature that could be used as evidence of close SMBH binary pairs at separations of $\sim 10^{-3} \mathrm{pc}$.

We assume that the accretion disk is as described by Shakura $\&$ Sunyaev (1973), i.e., an $\alpha$-disk. The $\alpha$-disk model is necessarily a mathematically convenient approximation rather than a fully physical model, but it is still a successful model in many 
respects for a wide variety of astrophysical accretion systems. Our results are not bound to the Shakura \& Sunyaev (1973) disk description, and we consider variations in Section 5. We require that (1) the azimuthally averaged disk midplane temperature is monotonic with radius in the inner $\sim 10^{3} R_{G}$, where $R_{G} \equiv G M c^{-2}$ and (2) $h / r$, the ratio of disk scale height to radius, is roughly constant. The recent considerations of azimuthal variations in disk temperature (Dexter \& Agol 2011; Dexter \& Quataert 2012) still assume that the scale of the variability does not change with radius so that for a given variability model, each azimuthally averaged annular emission is still only a function of $r$. A separate issue is that tidal interaction between the binary BHs and the gas disk can lead to spiral structures in either the gapped phase (Armitage \& Rice 2005) or the circumbinary phase (e.g., Rice et al. 2005; Cuadra et al. 2009). The spiral structure may be (but also may not be) averaged out in an azimuthal sense. For the sake of simplicity, we ignore any potential spiral structure in this paper. The secondary hole is assumed to have already found its way into the accretion disk of the primary and settled into a coplanar orbit, as has been shown to occur (Bogdanović et al. 2007; Dotti et al. 2010). As we discuss below, the mass ratios of interest are $q>0.003$. Below about $q=0.1-0.01$, a potential concern is that the mergers with halos containing small BHs will lead to a complete dispersal of the secondary halo before the $\mathrm{BH}$ can settle to the center. Recent numerical simulations, however, show that it is possible for mergers with dwarf satellites to lead to secondary BHs of mass ratios $q=0.01-0.001$ getting close enough to the primary for local processes to bring the BHs together (Bellovary et al. 2010). It is also possible that intermediate-mass BHs could form in situ in the accretion disk and would naturally be coplanar and have small mass ratios (McKernan et al. 2012).

\section{CONDITIONS FOR GAP OPENING}

There are two requirements that must be met in order for a gap in the disk to open: (1) the Hill sphere radius of the secondary must be larger than the disk scale height and (2) the gap closing timescale from the viscous reaction of the disk is longer than the gap opening timescale. Our calculations in this and the following section are inspired by and dependent upon the very closely analogous calculations for protoplanetary disks (e.g., Goldreich \& Tremaine 1980; Lin \& Papaloizou 1986; Artymowicz \& Lubow 1994; Takeuchi et al. 1996; Artymowicz \& Lubow 1996; Crida et al. 2006).

\subsection{Hill Sphere}

The Hill sphere defines the region around an orbiting body in which it is gravitationally dominant and has radius:

$$
R_{H} \equiv r\left(\frac{m_{s}}{3 M}\right)^{1 / 3}=r\left(\frac{q}{3}\right)^{1 / 3} .
$$

The secondary $\mathrm{BH}$ will clear a gap in the accretion disk if $R_{H} \geqslant h$, which happens only when $q \geqslant 3(h / r)^{3}$. In the gaspressure-dominated region where the binary orbits are most detectable, $h / r=0.004-0.008$ for thin disks. We take a conservative value of $h / r=0.01$, as our fiducial value implies that $q \geqslant 3 \times 10^{-6}$ and even minor mergers are sufficient to carve a gap of width $w \sim 2 R_{H}$.

\subsection{Gap Closing}

Gaps will only exist if the gravitational torques open them faster than viscous diffusion can close them. The timescale for opening a gap is

$$
t_{\mathrm{open}} \sim \frac{1}{\ell^{2} q^{2} \Omega}\left(\frac{w}{r}\right)^{2}
$$

where $\Omega$ is the angular frequency of the secondary and $\ell=$ $r \Omega c_{s}^{-1}$ is the order of the Lindblad resonance driving the gap creation (Takeuchi et al. 1996). The timescale to close the gap is

$$
t_{\text {close }} \sim \frac{w^{2}}{v}
$$

where $v$ is the kinematic viscosity. Requiring $t_{\text {close }}>t_{\text {open }}$, yields the requirement that

$$
q>\left(\frac{c_{s}}{r \Omega}\right)^{2} \alpha^{1 / 2}=\left(\frac{h}{r}\right)^{2} \alpha^{1 / 2},
$$

where $\Omega, c_{s}$, and $h$ are all taken to be at the radius of the secondary. This requirement is equivalent to $q>10^{-5}$ for our fiducial disk parameters. Alternative estimates to the critical mass ratio are possible (e.g., Lin \& Papaloizou 1986; Ward 1997; Crida et al. 2006; Kocsis et al. 2012a). For example, one may estimate the gap width by balancing angular momentum transfer rates via gravitational torques by the secondary on the disk with that via viscous diffusion from the disk. Then, requiring the gap width to be larger than the disk scale height or larger than the Hill sphere leads to estimates such as $q>A^{1 / 2}(h / r)^{5 / 2} \alpha^{1 / 2}$ or $q>(A / 3)(h / r)^{2} \alpha$, respectively, where $A \approx 50$ is a factor relating to the geometry and the sum of the torque modes derived from numerical simulations. Such calculations give comparable estimates.

\subsection{Longevity of the Gap}

In order for a gapped accretion disk to be of any observational consequence, it needs to persist for a reasonably large fraction of an AGN's lifetime. If the angular momentum transferred from the secondary to the outer disk is greater than the angular momentum transferred from the inner disk to the secondary, then the secondary and the gap will both migrate inward. The gap migration timescale when the local disk mass dominates over the secondary mass is (Takeuchi et al. 1996)

$$
t_{\text {migr }}=\frac{2}{3 \alpha}\left(\frac{h}{r}\right)^{-2} \Omega^{-1} .
$$

For a $10^{8} M_{\odot}$ primary with a secondary at $\sim 10^{3} R_{G}$, this corresponds to $\sim 3 \times 10^{5} \mathrm{yr}$. For secondaries with masses higher than our fiducial value, such that the secondary mass dominates over the local disk mass, the gap migration time is lengthened to $\sim 10^{6} \mathrm{yr}$, very similar to the timescale calculated by Haiman et al. (2009). Our timescale calculation neglects the fact that gas may accumulate at the outer edge of the gap, causing the gap width to decrease and potentially even close (Kocsis et al. $2012 \mathrm{a}, 2012 \mathrm{~b}$ ). If the gap does not close all the way, then the lifetime would be extended by a factor of $\sim 10$, though at reduced observability due to the reduced width for the fixed mass ratio.

When the BHs are close to each other, gravitational radiation is strong enough to shrink the orbit on interesting timescales. The Peters (1964) orbit-averaged evolution of the semimajor axis of two masses in orbit around each other with semimajor axis $a$ and eccentricity $e$ is

$$
\frac{d a}{d t}=-\frac{64 G^{3} M m_{s}\left(M+m_{s}\right)}{5 c^{5} a^{3}\left(1-e^{2}\right)^{7 / 2}}\left(1+\frac{73}{24} e^{2}+\frac{37}{96} e^{4}\right),
$$


where $G$ is the gravitational constant and $c$ is the speed of light. For the case we consider, $e=0$. The half-width of the gap is $R_{H}$, and the timescale for the secondary $\mathrm{BH}$ to move out of the gap is

$$
T_{g}=R_{H}\left(\frac{d a}{d t}\right)^{-1}=\frac{5 c^{5} a^{4}}{64 G^{3} M m_{s}\left(M+m_{s}\right)}\left(\frac{m_{s}}{3 M}\right)^{1 / 3} .
$$

Taking $a=r=10^{3} R_{G}$ and $q=10^{-2}$, then $T_{g} \approx 2 \times$ $10^{7}\left(M / 10^{8} M_{\odot}\right)$ yr, much longer than other relevant timescales.

In order to predict the actual rate of observable gapped disks, the actual rate of tight binary formation and their incidence with respect to the start of AGN activity are needed, but these are unknown. We can, however, predict the maximum fraction of an AGN's duration that a binary in an observable configuration can last, assuming that the binary companion of the right mass reaches an observable separation. The lifetime of an AGN is uncertain, but a typical value may be $\sim 10^{7} \mathrm{yr}$ (Martini 2004). For a gap lifetime of $\sim 10^{6} \mathrm{yr}$, up to $10^{-1}$ of a binary AGN's duty cycle can be in an observable gapped state. If binary acquisition and AGN activity are anti-correlated, then the implied observability would be much lower.

\section{EVOLUTION OF THE GAP}

Once a gap is established, it will evolve along three possible routes when considering only viscous effects. At the beginning, it is a gapped disk, effectively a standard disk with a missing annulus of width $w$. If the gap migration timescale is faster than or comparable to the timescale for the accretion of the disk material inside of the gap, then the gap will have constant $w / h$ with a decreasing radius.

If the mass accretion rate for the inner disk is faster than the gap migration rate, then it will partially decouple from the outer disk. This will first lead to an effective widening of the gap from the inner edge, making it more detectable. The inner disk will be continuously depleted and replaced by any material that accretes across the gap. If the mass accretion rate across the gap is the same as if there were no gap, then there would be no difference. On the other hand, if the accretion rate is a small fraction of what it would have been otherwise, then the inner disk would be replaced by an accretion disk with a smaller accretion rate.

The details of how much accretion will persist across a gap created by a satellite are the subject of current numerical simulations (e.g., Sesana et al. 2012; Shi et al. 2012; Noble et al. 2012). For the small mass ratios that are our primary interest here, however, it is plausible that the accretion rate is only reduced to $10 \%$ of what it would be in the absence of a satellite, i.e., $\dot{m}_{\text {red }}=0.1 \dot{m}$, where $\dot{m} \equiv \dot{M} / \dot{M}_{\text {Edd }}$ and $\dot{M}_{\text {Edd }} \equiv L_{\text {Edd }} \eta^{-1} c^{-2} \approx 3 \times 10^{-8}\left(M / M_{\odot}\right) M_{\odot} \mathrm{yr}^{-1}$ for an assumed $\eta=0.1$.

For higher mass ratios, it is plausible that very little matter will accrete past the gap, resulting in essentially no replacement of the inner gap. In such a case, instead of a gap, there would be a hole in the central accretion disk. The observational consequences of an accretion disk with a central hole-and the possible electromagnetic signal once the BHs merge-have been discussed before (Milosavljević \& Phinney 2005; O’Neill et al. 2009; Tanaka \& Menou 2010), and we return to this in Section 6.

The time to accrete the entire inner disk is

$$
t_{a}=M_{\mathrm{in}} \dot{M}^{-1}
$$

where $\dot{M}$ is the mass accretion rate and $M_{\text {in }}$ is the mass of the inner accretion disk. For our fiducial accretion disk, we assume the $\mathrm{BH}$ to be accreting at a fraction $\dot{m}=0.01$. The largest orbit we consider is roughly at the boundary outside of which free-free opacity dominates over Thomson scattering, so that the mass inside the gap is roughly

$$
M_{\text {in }} \approx 6 \times 10^{-11}\left(\frac{M}{M_{\odot}}\right)^{11 / 5} \alpha^{-4 / 5} \dot{m}^{23 / 15} M_{\odot}
$$

corresponding to an accretion timescale of

$$
t_{a} \approx 0.002 \alpha^{-4 / 5}\left(\frac{M}{M_{\odot}}\right)^{6 / 5} \dot{m}^{8 / 15} \mathrm{yr} .
$$

For our fiducial case, $t_{a} \approx 3 \times 10^{7} \mathrm{yr}$, so that the inner disk is depleting itself more slowly than the gap's inward migration. The above is only valid for $q \ll 1$. For mass ratios near unity, the dynamical torques will clear a hole inside of the orbit on a short timescale.

\section{SPECTRAL ENERGY DISTRIBUTION OF A GAPPED ACCRETION DISK}

The SED of a thermally emitting disk with a temperature that is only a function of the radius is

$$
F_{\lambda}(\lambda)=\int_{R_{\text {in }}}^{R_{\text {out }}} B_{\lambda}\left[T\left(r^{\prime}\right)\right] g\left(r^{\prime}\right) 2 \pi r^{\prime} d r^{\prime},
$$

where $B_{\lambda}(T)$ is the Planck function and $g\left(r^{\prime}\right)$ is a function that describes the emissivity as a function of radius. We assume that $g\left(r^{\prime}\right)=1$ (optically thick) where the disk material is present (i.e., $R_{\text {in }}<r^{\prime}<r-w$ and $r+w<r^{\prime}<R_{\text {out }}$ ) and $g(r)=0$ elsewhere. This assumption of an "on or off" emissivity is simplistic, and recent theoretical work (Tanaka et al. 2012; Sesana et al. 2012; Kocsis et al. 2012a) has examined in closer detail how the surface density changes close the gap. Kocsis et al. (2012a) found that close to the gap, the surface density will decrease outside of the Hill sphere. In most cases, the surface density does not drop all the way to zero, but it generally drops by over an order of magnitude. In the region of the disk immediately exterior to the gap, the surface density will increase to above the value it would have been in the absence of the disk. There is a similar enhancement in temperature because of the added pressure. Both of these effects of enhanced surface density and temperature are most prominent for high-mass ratios $(q>0.01)$ and small separations $\left(r<100 R_{G}\right)$. For the smaller mass ratios and wider separations that we consider here, this effect is small. Assuming gray body instead of blackbody emission, as we do, would tend to increase the observational signature (e.g., Tanaka $\&$ Menou 2010). Inverse-Compton scattering of the highest energy photons from the thermal spectrum will alter the SED, but this effect happens primarily at wavelengths shorter than $\sim 1000 \AA$ (Ross et al. 1992), meaning that it has little to no effect on the observability of gaps that we consider.

The absence of a ring in a thermally emitting disk will show up in the SED as a broad, shallow dip. Without a gap, the SED will fall off as $\lambda F_{\lambda} \sim \lambda^{-4 / 3}$. With a gap, the SED will appear roughly as a broken power law, with a slope that is steeper and shallower than $\lambda^{-4 / 3}$ blueward and redward of the maximum deviation from the standard SED. While it is obviously possible to model an observed SED with the proper Planck function 

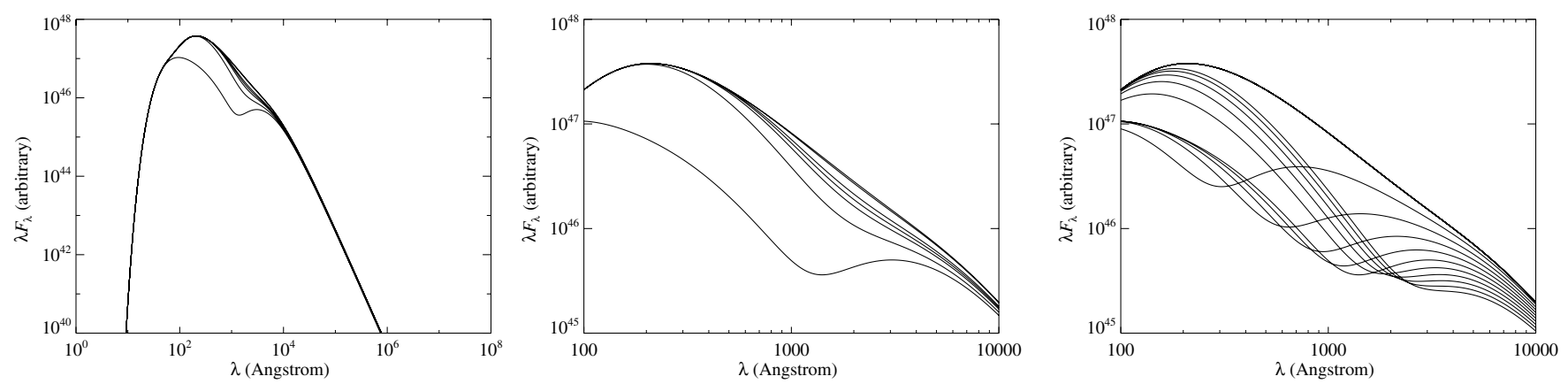

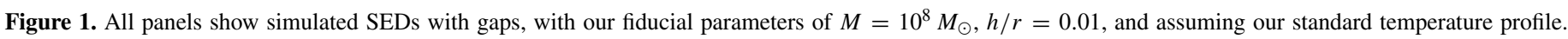

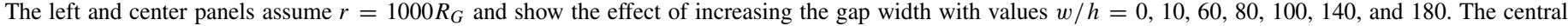

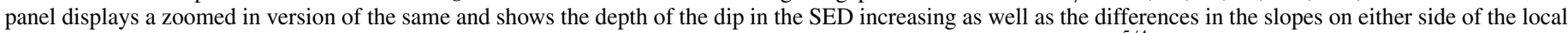

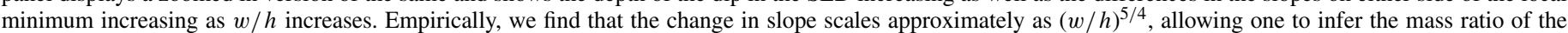

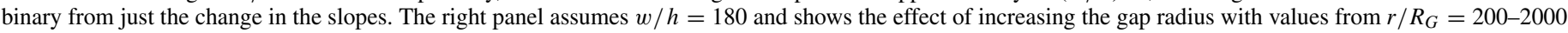

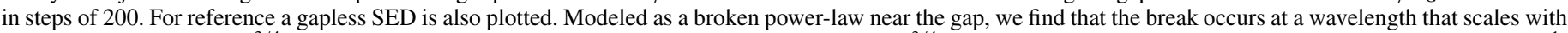

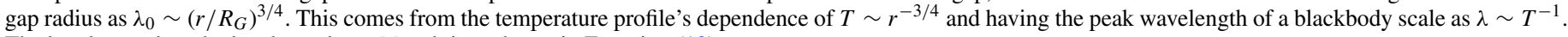
The break wavelength also depends on $M$ and $\dot{m}$ as shown in Equation (13).

treatment, we use a broken power-law model to demonstrate the precision required to identify a gapped disk. We generated SEDs (Figure 1) for gapped disks using Equation (11) for various values of $w, r / R_{G}, M$, and $\dot{m}$. For each simulated SED, we fitted a broken power law in the vicinity of the break of the form

$$
\lambda F_{\lambda}=\left\{\begin{array}{ll}
\lambda^{-x} & \lambda \leqslant \lambda_{0} \\
\lambda^{-y} & \lambda>\lambda_{0}
\end{array},\right.
$$

where $x>4 / 3$ and $y<4 / 3$ with continuity and an arbitrary normalization implied. The change in slope, $\Delta \equiv x-y$, from one side of the break to the other at $\lambda=\lambda_{0}$, increases as the gap width increases. We find

$$
\lambda_{0} \approx 140\left(\frac{r}{R_{G}}\right)^{3 / 4}\left(\frac{M}{10^{8} M_{\odot}}\right)^{1 / 4}\left(\frac{\dot{m}}{0.01}\right)^{-1 / 4} f(w / h) \AA \text {, }
$$

where $f(x)$ is a residual function that is smoothly varying over $x$ and has an absolute value $|f(w / h)-1|<0.35$ for most of the parameter space of interest. The dependence on $r, \dot{m}$, and $M$ is easily understood, since the peak wavelength of a blackbody scales as $\lambda \sim T^{-1}$ and temperature and mass scale as $T \sim \dot{m}^{1 / 4} r^{-3 / 4}$ in the outer regions where the secondary spends most of the time and $M \sim T^{-1 / 4}$. The dependence on $w$ comes from the area weighting of the output SED. The change in slope is well approximated by

$$
\Delta \approx 0.002(w / h)^{5 / 4}
$$

for $w / h<160$. Above $w / h>160$, the change in slope is larger than the above approximation because the emitting annulus just outside of the gap is further down the Wien exponential drop off. Since $w / h \sim q^{1 / 3}$ is purely a function of $q$ under our assumptions, it is possible to infer the mass ratio of the binary from just the change in slope, independent of $\dot{m}, M$, and $r$.

Syer \& Clarke (1995) and Rafikov (2012) found that a gapped accretion disk would have an SED such that $\lambda F_{\lambda} \propto \lambda^{12 / 7}$. If $T(r) \sim r^{-0.9}$, then the peak of the SED scales as $\lambda F_{\lambda} \propto \lambda^{-1.7}$, approximately as $\lambda^{12 / 7}$. There is no qualitative difference in the approach to modeling SEDs of such gapped disks. We find that the change in the power-law slope for such a temperature power law only decreases by about $10 \%$ compared to our fiducial temperature profile. Thus, one could use the overall slope of the continuum power law to identify gapped accretion disks and then use detailed measurements of $\Delta$ and $\lambda_{0}$ to infer properties of the BHs.

\section{SED OF AN ACCRETION DISK WITH A HOLE}

For higher mass ratios and suitably small separations, almost the entire region inside of the orbit will be dynamically unstable, as can be seen from the size of the Roche lobes for $q \approx 1$ binaries. Thus, all gas will be cleared out of this region, and if the gas cannot be replaced, then it will leave an accretion disk with a hole of size $r \sim 2 a$ in the center. Like accretion across the gap, the ability or inability of the disk to accrete a significant amount of matter into the hole and the timescale on which it does so is a matter of debate (e.g., Artymowicz \& Lubow 1994, 1996; Milosavljević \& Phinney 2005; Hayasaki et al. 2007; Cuadra et al. 2009; Tanaka \& Menou 2010; Shapiro 2010; Sesana et al. 2012; Shi et al. 2012; Noble et al. 2012). Because of resonant interactions between the binary and the gas disk, the orbit of the secondary can become eccentric_-particularly when starting with a substantial eccentricity - so that accretion onto the secondary is non-negligible (Roedig et al. 2011). Assuming that the inner hole is mostly cleared of continuum-emitting material and neglecting the uncertain amount of accretion onto the secondary, the effect of an inner hole on the SED is much more dramatic than that of a gap. Most or all of the shortest wavelength emission will be cut out, leaving a red SED.

The absence of an inner accretion disk owing to a BH merger event may therefore be identified through the broadband SED. The outer accretion disk will still have a high accretion rate, and should be luminous in optical bands. Indeed, the outer disk may be abnormally luminous in optical, or even in UV bands (see, e.g., Kocsis et al. 2012b), depending on the mass accretion rate and the truncation radius. Considered simply, UV luminosity is not a good diagnostic: a holed disk around a $\mathrm{BH}$ of lower mass is observationally degenerate with a filled inner disk around a $\mathrm{BH}$ of higher mass. Rather, diminished X-ray emission is a better diagnostic, since non-thermal X-ray emission is thought to tap into the deep gravitational potential close to the $\mathrm{BH}$ (Tanaka et al. 2012). In SMBHs, most X-ray emission arises from inverse-Compton scattering of lower energy photons off hot electrons in a corona of which relatively little is known. It is possible that an X-ray faint AGN could be caused by the absence or variability of the hot corona (Miniutti et al. 2012), but this requires UV observations to show that there are thermal disk photons in the UV available to be inverseCompton scattered (e.g., Leighly et al. 2007b). The amount of $\mathrm{X}$-ray diminishing, however, depends critically on how much 
accretion through the hole is diminished, which has been found in some studies to be altered only very mildly (Sesana et al. 2012). If accretion is greatly diminished, then residual accretion within the hole may persist at a low level, but even this gives rise to X-ray variability in Sgr A*. Thus, X-ray variability may be an additional hallmark, which has been investigated in detail by surveys such as the extended Roentgen Survey with an Imaging Telescope Array (Sesana et al. 2012). Thus, a source that is luminous in optical bands but intrinsically faint (not faint due to obscuration) and potentially variable in X-rays might signal a recent or pending $\mathrm{BH}$ merger event, including potential electromagnetic counterparts to gravitational wave detections from pulsar timing array experiments (Tanaka et al. 2012).

It is possible that such sources have already been identified. When the distance to a given AGN is known a priori, e.g., through spectroscopy, Sloan Digital Sky Survey (SDSS) colors are able to distinguish whether or not a source is intrinsically luminous in, e.g., red bands, or is apparently only red through obscuration. In particular, $g-i$ is very effective, or $\Delta(g-i)$, where one measures color relative to the mean quasar color in a given redshift bin. Hall et al. (2006) employed this technique to 3814 SDSS quasars with the intention of isolating obscured BHs. Follow-up observations with Chandra revealed that a subset of intrinsically red quasars are not X-ray-faint because of obscuration. In particular, seven sources show no evidence of even moderate intrinsic absorption in X-rays, and two are constrained to have internal column densities below $2 \times 10^{21} \mathrm{~cm}^{-2}$ (Hall et al. 2006). This suggests that such sources are rare $\left(7 / 3814=1.8 \times 10^{-3}\right)$, consistent with expectations based on the peculiar configuration required and the relevant timescales, and also consistent with expectations based on broader surveys (Gibson et al. 2008).

An interesting example of a quasar that is optically very luminous but X-ray faint is PHL 1811, but because of the highquality UV data of this source (Leighly et al. 2007a) and restframe UV observations of high-redshift analogs (Wu et al. 2011; Lane et al. 2011), detailed models of the SED show it to have a thermally emitting disk toward $\sim 6 R_{G}$, thus lacking an inner hole (Leighly et al. 2007b). This example illustrates that Xray faintness is not a sufficient condition to infer an accretion disk hole, but it is probably a necessary condition. The most promising method for finding holey accretion disks is to select $\mathrm{X}$-ray-faint quasars for full SEDs or spectral modeling in order to establish the amount of UV and X-ray absorption as well as a rough estimate of the size of the inner edge of the accretion disk. For example, SDSS J094533.99+100950.1 has only upper limits on its X-ray flux (Wu et al. 2011) and very faint UV-continuum emission (Czerny et al. 2011), but detailed modeling of the broadband SED and UV spectrum revealed that it is, depending on the extinction model assumed, heavily extincted, with an accretion disk extending into at least $\sim 10 G M c^{-2}$ (Czerny et al. 2011).

\section{DISCUSSION AND CONCLUSIONS}

We have described the basic observational appearance of a $\mathrm{BH}$ with a gapped or holed accretion disk, both of which are observable through their broadband SEDs. We have focused our consideration on parameter space that results in observable signatures between $2000 \AA$ and $2 \mu \mathrm{m}$. At shorter wavelengths, absorption of ultraviolet photons will prevent unambiguous determination of details of the AGN continuum. At longer wavelengths, reprocessed emission from dust grains will similarly contaminate thermal emission from an accretion disk. In this range, however, it is possible to measure the continuum emission to the precision needed to infer the presence of a gap. Current and future large surveys such as SDSS, Pan-Starrs, and LSST may be able to exploit the observational signatures we have developed.

The primary outcome of this paper is to define clear diagnostics of accretion disks with gaps and holes. To do so, we have made some simplifying assumptions that allow for the clearest picture of what will happen to the accretion disk SED, but there are, of course, some potential complications that will need to be considered in the future. One such complication is that we have treated the emission as only coming from the top of the accretion disk. Emission coming from the walls of the gap will complicate the signal and, depending on the vertical temperature structure, either increase or decrease the observability of the gap, potentially by a large amount given the possibility of dramatically increased $h / r$ at the outer boundary from gas pile up (Kocsis et al. 2012a). Tidal features produced by the secondary as seen in simulations of protoplanetary disks will also need to be considered and may lead to additional corroborating observational signals. The secondaries that are observable have periods of months to several years, allowing for a potential detection of a variable or modulating signal (Tanaka et al. 2010; Tanaka \& Menou 2010; Sesana et al. 2012; Noble et al. 2012). One such periodic signature from an accreting secondary could be Fe Xxv and Fe XXVI narrow emission lines that would move with the secondary. Such a measurement should be possible with the Astro-H X-ray observatory (Takahashi et al. 2010).

Another potential complication is that the population of observed quasar SEDs has a large variance (e.g., Richards et al. 2006; Shang et al. 2011) that arises from a number of unknown causes not thought to be connected to the occurrence of any secondary BH. For example, typical optical-to-X-ray spectral indices $\left(F_{v} \propto v^{\alpha_{\mathrm{ox}}}\right)$ can range from $\alpha=-1.8$ to -1.2 . Unambiguously discerning between low X-ray activity arising from quasars coming from the low end of a natural range and low X-ray activity arising from quasars with a missing inner disk requires sufficiently deep X-ray observations. Regarding gapped accretion disks, our focus on local deviations from an overall power-law continuum emission mitigates the potential confusion from variance in SEDs arising from other causes. That is, it is possible to discern a dip in a power-law SED portion without a priori knowing what the power-law slope is. A full theoretical understanding of normal quasar continuum emission would, of course, allow one to model gaps in accretion disks with utmost fidelity.

We thank Cole Miller and Mike Eracleous for extremely useful discussions as well as Zoltan Haiman, Bence Kocsis, Mateusz Ruszkowski, Alberto Sesana, and Taka Tanaka for thoughtful comments. We also thank Jim Saborio and staff for their hospitality during which a large portion of this work was completed. K.G. acknowledges support provided by NASA through Chandra Awards GO0-11151X and G02-13111X and through Hubble Award HST-GO-12557.01-A awarded by the STScI.

\section{REFERENCES}

Armitage, P. J., \& Rice, W. K. M. 2005, arXiv:astro-ph/0507492

Artymowicz, P., \& Lubow, S. H. 1994, ApJ, 421, 651

Artymowicz, P., \& Lubow, S. H. 1996, ApJ, 467, L77 
Baker, J. G., Boggs, W. D., Centrella, J., et al. 2008, ApJ, 682, L29 Begelman, M. C., Blandford, R. D., \& Rees, M. J. 1980, Nature, 287, 307 Bellovary, J. M., Governato, F., Quinn, T. R., et al. 2010, ApJ, 721, L148

Blecha, L., Loeb, A., \& Narayan, R. 2012, arXiv:1201.1904

Bogdanović, T., Reynolds, C. S., \& Miller, M. C. 2007, ApJ, 661, L147

Boroson, T. A., \& Lauer, T. R. 2009, Nature, 458, 53

Chang, P., Strubbe, L. E., Menou, K., \& Quataert, E. 2010, MNRAS, 407, 2007

Comerford, J. M., Gerke, B. F., Stern, D., et al. 2012, ApJ, 753, 42

Crida, A., Morbidelli, A., \& Masset, F. 2006, Icarus, 181, 587

Cuadra, J., Armitage, P. J., Alexander, R. D., \& Begelman, M. C. 2009, MNRAS, 393, 1423

Czerny, B., Hryniewicz, K., Nikołajuk, M., \& Sądowski, A. 2011, MNRAS, 415,2942

Dexter, J., \& Agol, E. 2011, ApJ, 727, L24

Dexter, J., \& Quataert, E. 2012, MNRAS, 426, L71

Dotti, M., Volonteri, M., Perego, A., et al. 2010, MNRAS, 402, 682

Eracleous, M., Boroson, T. A., Halpern, J. P., \& Liu, J. 2012, ApJS, 201, 23

Espaillat, C., Calvet, N., Luhman, K. L., Muzerolle, J., \& D’Alessio, P. 2008, ApJ, 682, L125

Gaskell, C. M. 1996, ApJ, 464, L107

Gibson, R. R., Brandt, W. N., \& Schneider, D. P. 2008, ApJ, 685, 773

Goldreich, P., \& Tremaine, S. 1980, ApJ, 241, 425

Haiman, Z., Kocsis, B., \& Menou, K. 2009, ApJ, 700, 1952

Hall, P. B., Gallagher, S. C., Richards, G. T., et al. 2006, AJ, 132, 1977

Hayasaki, K., Mineshige, S., \& Sudou, H. 2007, PASJ, 59, 427

Jahnke, K., \& Macciò, A. V. 2011, ApJ, 734, 92

Kocsis, B., Haiman, Z., \& Loeb, A. 2012a, arXiv:1205.4714

Kocsis, B., Haiman, Z., \& Loeb, A. 2012b, arXiv: 1205.5268

Lane, R. A., Shemmer, O., Diamond-Stanic, A. M., et al. 2011, ApJ, 743, 163

Leighly, K. M., Halpern, J. P., Jenkins, E. B., \& Casebeer, D. 2007a, ApJS, 173,1

Leighly, K. M., Halpern, J. P., Jenkins, E. B., et al. 2007b, ApJ, 663, 103

Lin, D. N. C., \& Papaloizou, J. 1986, ApJ, 309, 846

Martini, P. 2004, in Coevolution of Black Holes and Galaxies, ed. L. C. Ho (Cambridge: Cambridge Univ. Press), 169
McKernan, B., Ford, K. E. S., Lyra, W., \& Perets, H. B. 2012, MNRAS, 425,460

Merritt, D., Milosavljević, M., Favata, M., Hughes, S. A., \& Holz, D. E. 2004, ApJ, 607, L9

Milosavljević, M., \& Merritt, D. 2003, ApJ, 596, 860

Milosavljević, M., \& Phinney, E. S. 2005, ApJ, 622, L93

Miniutti, G., Brandt, W. N., Schneider, D. P., et al. 2012, MNRAS, 425, 1718

Noble, S. C., Mundim, B. C., Nakano, H., et al. 2012, ApJ, 755, 51

O’Neill, S. M., Miller, M. C., Bogdanović, T., Reynolds, C. S., \& Schnittman, J. D. 2009, ApJ, 700, 859

Peters, P. C. 1964, Phys. Rev., 136, 1224

Rafikov, R. R. 2012, arXiv:1205.5017

Rice, W. K. M., Lodato, G., \& Armitage, P. J. 2005, MNRAS, 364, L56

Richards, G. T., Lacy, M., Storrie-Lombardi, L. J., et al. 2006, ApJS, 166, 470

Richstone, D., Ajhar, E. A., Bender, R., et al. 1998, Nature, 395, A14

Roedig, C., Dotti, M., Sesana, A., Cuadra, J., \& Colpi, M. 2011, MNRAS, 415, 3033

Ross, R. R., Fabian, A. C., \& Mineshige, S. 1992, MNRAS, 258, 189

Sesana, A., Roedig, C., Reynolds, M. T., \& Dotti, M. 2012, MNRAS, 420, 860

Shakura, N. I., \& Sunyaev, R. A. 1973, A\&A, 24, 337

Shang, Z., Brotherton, M. S., Wills, B. J., et al. 2011, ApJS, 196, 2

Shapiro, S. L. 2010, Phys. Rev. D, 81, 024019

Shi, J.-M., Krolik, J. H., Lubow, S. H., \& Hawley, J. F. 2012, ApJ, 749, 118

Smith, K. L., Shields, G. A., Bonning, E. W., et al. 2010, ApJ, 716, 866

Syer, D., \& Clarke, C. J. 1995, MNRAS, 277, 758

Syer, D., Clarke, C. J., \& Rees, M. J. 1991, MNRAS, 250, 505

Takahashi, T., Mitsuda, K., Kelley, R., et al. 2010, Proc. SPIE, 7732, $77320 Z$

Takeuchi, T., Miyama, S. M., \& Lin, D. N. C. 1996, ApJ, 460, 832

Tanaka, T., Haiman, Z., \& Menou, K. 2010, AJ, 140, 642

Tanaka, T., \& Menou, K. 2010, ApJ, 714, 404

Tanaka, T., Menou, K., \& Haiman, Z. 2012, MNRAS, 420, 705

Volonteri, M., Haardt, F., \& Gültekin, K. 2008, MNRAS, 384, 1387

Volonteri, M., Haardt, F., \& Madau, P. 2003, ApJ, 582, 559

Ward, W. R. 1997, Icarus, 126, 261

Wu, J., Brandt, W. N., Hall, P. B., et al. 2011, ApJ, 736, 28 